\title{
Photocontrol of Polypeptide Membrane Structure and Functions by cis-trans Isomerization in Side- Chain Azobenzenesulfonate Groups
}

\author{
Morimasa SATo, Takatoshi KinOSHITA, ${ }^{*}$ Akira TAKIZAWA, \\ Yoshiharu TsUJITA, and Toshihiko OSADA \\ Department of Material Science \& Engineering, Nagoya Institute of Technology, \\ Gokiso-cho, Showa-ku, Nagoya 466, Japan
}

(Received Octob'er 11, 1988)

\begin{abstract}
Photoresponsive polypeptides, poly(L-glutamic acid) containing 1.9, 14.1, 46.3 $\mathrm{mol} \%$ azobenzenesulfonate moieties in the side chains (azo-S-PGA), have been prepared by the condensation reaction of poly(L-glutamic acid) (PGA, $M_{v}=1.19 \times 10^{5}$ ) with 4-amino-1,1'azobenzene- $4^{\prime}$-sulfonic acid sodium salt in dimethylformamide (DMF) solution and the membranes were obtained by casting their DMF solution with 4,4'-diaminodiphenylmethane as a cross-linking agent. trans to cis Isomerization of the azobenzenesulfonate moieties induced by ultraviolet (UV) light resulted in $\alpha$-helix to coil transition in the membrane composed of azo.S-PGA containing 14.1 $\mathrm{mol} \%$ azobenzenesulfonate moieties at adequate $\mathrm{pHs}$, indicating the electrostatic repulsion between the sulfonate anions in the azo photochromic side chains and the neighboring glutamate anions to be enhanced by photoinduced changes in the geometry of the azo chromophores. As a result, the membrane potentials of azo.S-PGA membrane containing $14.1 \mathrm{~mol} \%$ azobenzenesulfonate moieties were strongly dependent on UV light irradiation. It was also found that photoinduced changes of the membrane structure and membrane potentials were irreversible. On the other hand, the structure of the azo.S-PGA membrane containing 1.9 and $46.3 \mathrm{~mol} \%$ azobenzenesulfonate moieties was shown to be independent of UV light irradiation at any $\mathrm{pH}$. The former ineffectiveness of the light irradiation was attributed to small amounts of azo photochromic moieties and the latter to the fact that the azo-S-PGA membranes with large amounts of azobenzenesulfonate moieties are in random coil structures at any $\mathrm{pH}$ even in the drk.
\end{abstract}

KEY WORDS Photoresponsive Polypeptide / Azobenzenesulfonate Side Chain / Membrane / Photoinduced Conformational Change / Photoinduced Membrane Potential Change /

It has been shown, as an example of the photoregulated function of artificial membranes, that the photochemical reaction of photochromic molecules, such as azobenzene, spiropyran and other dyes, entrapped in an inert membrane matrix can induce significant changes in membrane potentials across cellulose $^{1-4}$ and poly(vinyl chloride) ${ }^{5-8}$ membranes. It has been reported, furthermore, that the membrane potential can be also photoregulated by using photoresponsive polymers having such photochromic molecules incorporated as pendant groups in the polymer chains. For example, Irie et al., ${ }^{9}$ showed that visible light irradiation induced reversible changes in the potential across a membrane composed of poly(methyacrylic acid) containing spirobenzopyran moieties based on the photoinduced conformational change of the polymer chain in a high ionic concentration range and photoinduced decrease in the negative fixed charge density of the membrane in the low ionic

* To whom corresponding should be addressed. 
concentration range, respectively. Both were derived from the photoisomerization of spirobenzopyran pendant groups. Understanding the mechanism of photoinduced changes in the interactions in the photoresponsive polymer between the photochromic moiety and the remaining groups, closely linked to the conformational and functional responses, may give a possible extension of the application of photoresponsive polymers, in addition to molecular level insight into the behavior of natural photo-receptors.

Recently, much effort has been made for the characterization of the photoresponsive properties of polypeptides ${ }^{10-14}$ with azobenzene side chains, since they may serve as effective photosensitizers based on conformational variations. Most of these studies, however, have been limited to the polypeptides in solution.

$\mathrm{We}^{15-20}$ have already studied the photoresponsive behavior of the polypeptides in the membrane system and showed the photocontrol of membrane structure ${ }^{18,19}$ and functions, such as permeabilities ${ }^{16-20}$ and membrane potentials, ${ }^{15,17-20}$ of poly(L-glutamic acid) (PGA) containing azobenzene and pararosaniline groups, respectively, in the side chains owing to cooperative effect between the photoreaction of the photochromic side chain and acid dissociation of glutamic acid side chain in the membrane.

We report here the photoinduced conformational transition and membrane potential changes of a membrane composed of PGA containing azobenzenesulfonate groups in the side chains (azo-S-X-PGA, $\mathrm{X}$ is a $\mathrm{mol} \%$ of azobenzenesulfonate groups). Photoinduced geometrical change of the azobenzenesulfonate moieties from trans to cis forms resulted in $\alpha$-helix to coil transition of azo-S-14.1-PGA membrane at adequate $\mathrm{pHs}$ based on photoinduced increase in electrostatic repulsion between anionic azosulfonate and neighboring glutamic acid side chains around the $\alpha$-helical backborn. Ultra-violet light irradiation to the azo.S-14.1-PGA membrane also induced de- $\alpha$-helix

random coil

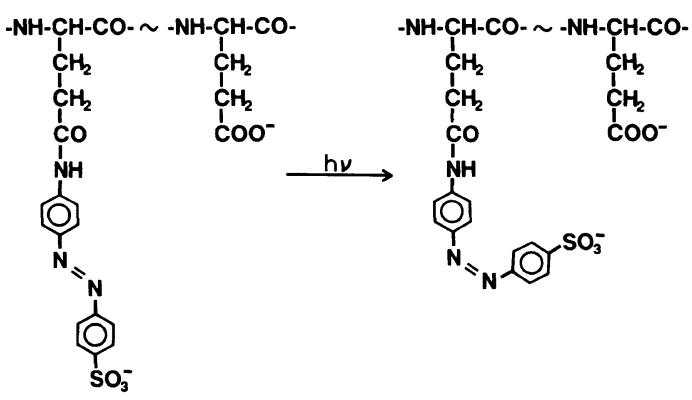

Scheme 1.

crease in the membrane potential based on decrease in the effective charge density of the membrane resulting from the induced $\alpha$-helix to coil transition of the membrane.

This is the first example of photoinduced conformational changes of polypeptide membrane system using azobenzene moiety as a photochromic group. The photoresponsive behavior of azo.S-PGA in aqueous solution ${ }^{21}$ and azo $\cdot$ S-PGA adsorbed porous membrane ${ }^{22}$ is reported elsewhere.

\section{EXPERIMENTAL}

\section{Materials}

Poly(L-glutamic acid) (PGA, $M_{v}=1.19 \times$ $10^{5}$ ) containing azobenzenesulfonate moieties in the side chains (azo-S-X-PGA, $\mathrm{X}$ is a $\mathrm{mol} \%$ of the azobenzenesulfonate moieties) was synthesized by a coupling reaction between PGA and 4-amino-1,1'-azobenzene-4'-sulfonic acid sodium salt (azo-S). The details of the preparation of the polypeptide were previously reported. ${ }^{21}$

Azo.S-PGAs were dissolved in dimethylformamide (DMF) followed by $10 \mathrm{~mol}^{\%} \%$ of 1-ethyl-3,3-dimethylaminopropylcarbodiimide hydrochloride(WSC), $N$-hydroxybenzotriazole $(\mathrm{HOBt})$ and of 4,4'-diaminodiphenyl methane for the introduction of a cross-linkage into the membrane, respectively (Scheme 2 ). The solution was pored onto a glass plate and allowed to evapolate at $40^{\circ} \mathrm{C}$. The mem- 


\section{Photocontrol of Polypeptide Membrane Structure and Function}

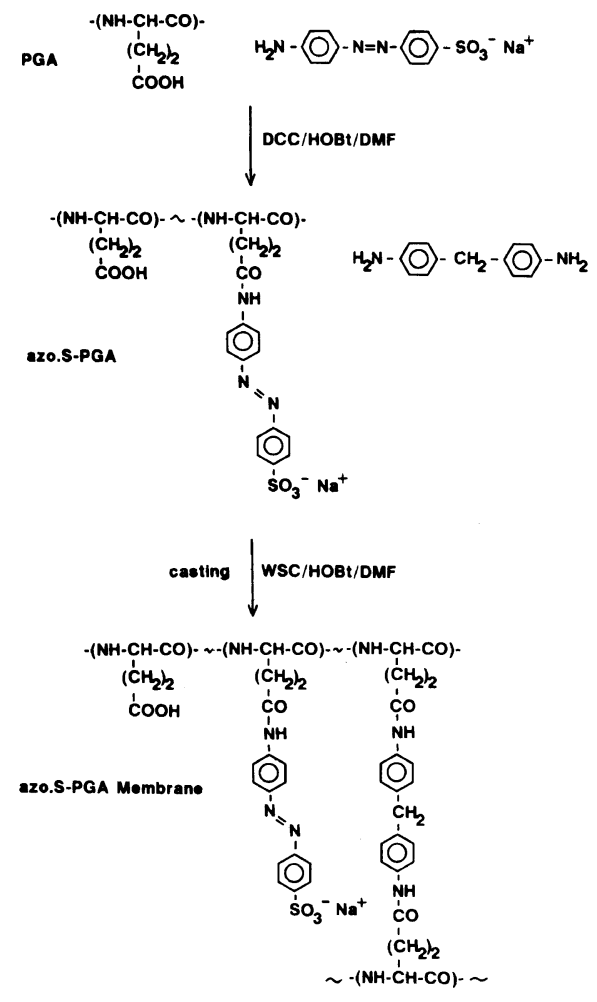

Scheme 2.

brane obtained was washed with methanol for several times and dried in vacuo. The azo-S-PGA membrane was not dissolved in DMF or alkaline aqueous solution, indicating the formation of a cross-linkage in the azo.S-PGA membrane as shown in Scheme 2.

\section{Measurements}

Absorption spectra and circular dichroism (CD) spectra were recorded by JASCO UVIDEC 670 spectrophotometer and JASCO J-40C spectropolarimeter, respectively. The molar ellipticity, $[\theta]$, of the membrane was calculated by

$$
[\theta]=\frac{\theta \times M_{w}}{d \times l}
$$

where $\theta, M_{w}, d$, and $l$ are ellipticity, average residual molecular weight, density and membrane thickness, respectively.

The method for measurement of the mem- brane potential was similar to that previously reported. ${ }^{17,20}$ The potential of the higher concentration side $\left(c_{1}\right)$ was taken as zero. The $\mathrm{KCl}$ concentration ratio, $c_{1} / c_{2}$, was fixed at 10 . Membrane potential measurements were performed at $25^{\circ} \mathrm{C}$.

The degree of hydration of the membrane, $H$, weight fraction of water in the waterswollen membrane, was measured at $25^{\circ} \mathrm{C}$ by the similar manner as previously reported. ${ }^{17,20}$

Irradiation was carried out with a $500 \mathrm{~W}$ super-high pressure mercury lamp (USHIO UVD-500) equipped Toshiba UVD-33S glass filter for ultraviolet light unirradiation $(250 \mathrm{~nm}<\lambda<380 \mathrm{~nm})$.

For all measurement, azo-S-PGA membranes were kept in the dark for a few days to ensure that all of azo groups would be in the trans form at the beginning of measurement.

\section{RESULTS AND DISCUSSION}

\section{Conformation of Dark-Adapted Azo $S$-PGA Membrane \\ The conformation of azo.S-PGA mem-} branes in the dark was examined by circular dichroism (CD) measurements. $\mathrm{We}^{21,22}$ have already shown that, with a solution system, azo-S-PGAs containing 1.9, 9.3, and 14.1 $\mathrm{mol} \%$ azobenzenesulfonate moieties change their backborn structures from the right handed $\alpha$-helix in acid solution to the random coil by increasing the $\mathrm{pH}$ of the aqueous solution. It was also shown, however, that azo.SPGA containing a large amount of azobenzenesulfonate moiety, $46.3 \mathrm{~mol} \%$, was always in the random coil structure in a wide range of $\mathrm{pH}$ because of the decrease in stability of the $\alpha$ helix structure via electrostatic repulsion between side chain sulfonate anions. A similar result was obtained with their membrane systems. Figure 1 shows the $\mathrm{pH}$ dependence of molar ellipticity of the CD band at $222 \mathrm{~nm}$, $[\theta]_{222}$ associated with the $\alpha$-helix content of the polypeptide, of azo-S-PGA membrane containing $46.3,14.1$, and $1.9 \mathrm{~mol} \%$ azobenzene- 
(a)

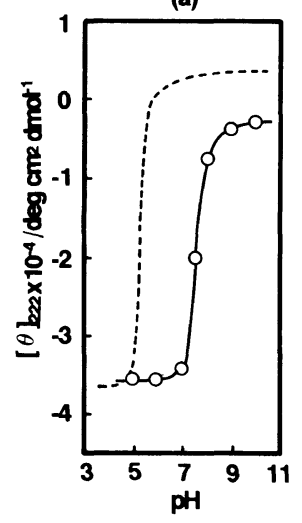

(b)

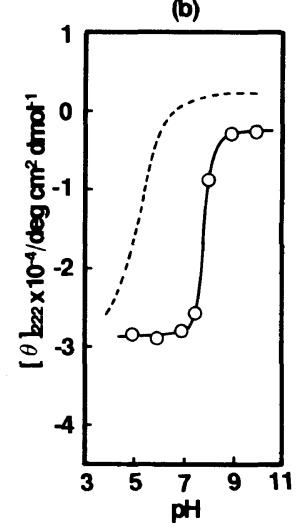

(c)

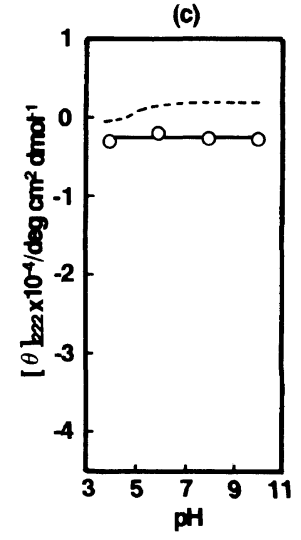

Figure 1. $\mathrm{pH}$ dependence of molar ellipticity at $222 \mathrm{~nm},[\theta]_{222}$, of the membrane composed of poly $(\mathrm{L}-$ glutamic acid) containing: (a), 1.9; (b), 14.1; and (c) $46.3 \mathrm{~mol} \%$ azobenzenesulfonate groups in the side chains in the dark at $25^{\circ} \mathrm{C}$. The dotted lines are for aqueous solutions of the same systems.

sulfonate moieties. Azo -S-1.9-PGA and azo-S14.1-PGA membranes exhibited a typical helix to coil transition by increasing the $\mathrm{pH}$ of the aqueous solution (Figure 1(a) and (b)). The dotted lines in Figure 1(a) and (b) show $\mathrm{pH}$ dependence of $[\theta]_{222}$ for aqueous solution systems of azo.S-1.9-PGA and azo.S-14.1-PGA, respectively. It is clear that the $\mathrm{pH}$ of the helix to coil transition point for both membrane systems significantly shifted to the higher $\mathrm{pH}$ value compared with that for solution systems. This suggests a decrease in the degree of dissociation of L-glutamic acid moiety in the membrane because of the higher degree of hydrophobicity in the membrane base on the azobenzene and cross-linking diphenyl methane moieties and the lower hydration activity of water in the membrane. On the other hand, $[\theta]_{222}$ of azo-S-46.3-PGA membrane (Figure 1(c)) was shown to be small and almost independent on $\mathrm{pH}$, i.e., also with the membrane system, a large amount of side chain azo sulfonate anions, with pKa below 1.0, could decrease the stability of the helix because of electrostatic repulsion even in acid solution in the dark.

\section{Photoinduced Conformational Changes of Azo $\cdot S$-PGA Membrane}

Figure 2(a) shows photo-induced changes in

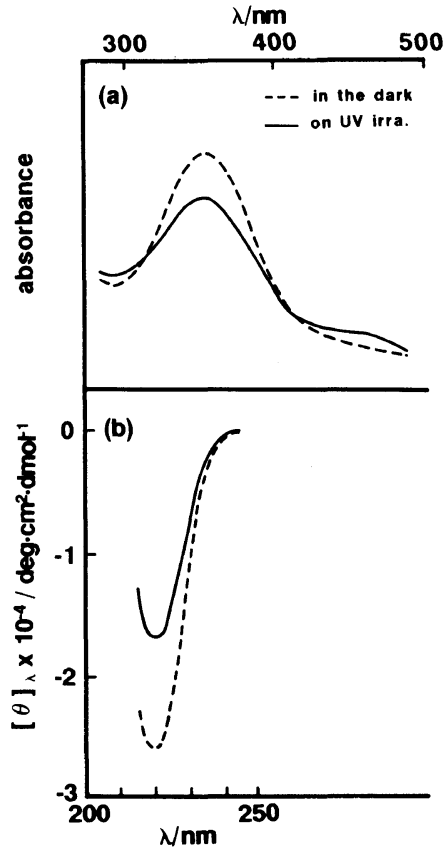

Figure 2. (a) Photoinduced change in absorption spectra of the membrane composed of poly(L-glutamic acid) containing $14.1 \mathrm{~mol} \%$ azobenzenesulfonate groups in the side chains at $\mathrm{pH} 7.5$ and at $25^{\circ} \mathrm{C}$. (b) Photoinduced change in CD spectra of the membrane composed of poly(L-glutamic acid) containing $14.1 \mathrm{~mol} \%$ azobenzenesulfonate groups in the side chains at $\mathrm{pH} 7.5$ and at $25^{\circ} \mathrm{C}$.

the absorption spectra of an azo -S-PGA membrane containing $14.1 \mathrm{~mol}^{\%} \%$ azobenzenesulfonate moieties $(\mathrm{azo} \cdot \mathrm{S}-14.1-\mathrm{PGA})$ in aqueous 
solution at $\mathrm{pH} 7.5$. The main absorption band at $355 \mathrm{~nm}$ decreased by UV irradiation $(250$ $\mathrm{nm}<\lambda<380 \mathrm{~nm}$ ), indicating that the azobenzenesulfonate moieties in the side chains can be isomerized from the trans to the cis forms by irradiation. The photoconversion of this isomerization was calculated from the molar extinction coefficient of the trans isomer $\left(\varepsilon_{\text {trans }}=2.59 \times 10^{4}\right)$ of azo-S-PGA, assuming that the molar extinction coefficient of the all-cis isomer is negligible. The photoconversion of this isomerization obtained was $c a$. $55 \%$ at $\mathrm{pH} 7.5$, which is the same as that obtained with a solution system of azo $\cdot \mathrm{S}-14.1$ PGA. ${ }^{21}$ The opposite isomerization from the cis to the trans form was achieved when the membrane was kept in the dark.

The effects of light irradiation $(250 \mathrm{~nm}<$ $\lambda<380 \mathrm{~nm}$ ) on the structure of the membranes were influenced by the amounts of azobenzenesulfonate moieties. Irradiation of azo $\mathrm{S}$ 1.9-PGA and azo-S-46.3-PGA membranes did not induce any structural changes in the membranes at any $\mathrm{pH}$. The former ineffectiveness of the light irradiation may be attributed to the small amounts of azophotochromic moieties (azo-S-1.9-PGA) and the latter one to no variation in the conformation at any $\mathrm{pH}$ (azo-S-46.3-PGA) even in the dark (Figure 1(c)). In contrast, the azo $\cdot$ S-14.1-PGA membrane exhibited remarkable conformational changes upon irradiation at adequate $\mathrm{pH}$. Figure 2(b) shows photoinduced changes in CD spectra of azo.S-14.1-PGA membrane at $\mathrm{pH}$ 7.5. The negative band at $222 \mathrm{~nm}$ connected with the $\alpha$-helix structure is shown to decrease by irradiation. As a result, it can be estimated that the azo-S-14.1-PGA membrane changes the helix content from $76 \%$ to $46 \%$ at pH 7.5 by light. Ciardelli et al. ${ }^{10}$ reported the $\alpha$-helix structure of PGA containing up to 16 $\mathrm{mol} \%$ azobenzene groups (without sulfonate anions) in the side chains in the solution system to be independent of the ultra-violet light irradiation at any $\mathrm{pH}$. It should be emphasized, therefore, that increase in the photo- sensitivity of the azo $\cdot \mathrm{S}-14.1$-PGA membrane is due to the introduction of the sulfonate anions to the azo photochromic moieties. Figure 3 shows variations in $[\theta]_{222}$ associated with photoinduced conformational changes of azo $\cdot \mathrm{S}-14.1-\mathrm{PGA}$ membrane, $\Delta[\theta]_{222}=[\theta]_{222}$ on UV irradiation $-[\theta]_{222}$ in the dark, at various $\mathrm{pH}$. It is clear that there were no effects by irradiation below pH 5.0 where the neighboring L-glutamic acid moieties are in the undissociated state in the membrane and at higher pHs (>9.0) where the polypeptide is in a random coil in the dark. As a result, the copolypeptide membrane was shown to have a relatively narrow $\mathrm{pH}$ range where the photoinduced conformational transition occurs. These results establish that the UV light-induced remarkable conformational change of the membrane arises from an additional increase in the local charge density of the environment

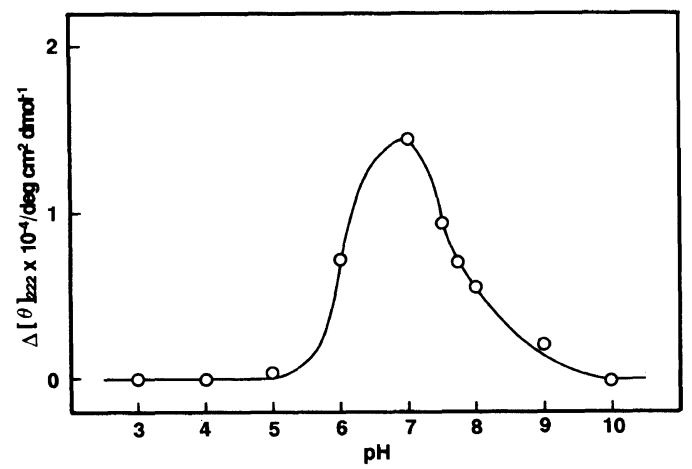

Figure 3. $\mathrm{pH}$ dependence of $\Delta[\theta]_{222}\left(=[\theta]_{222}\right.$ on UV irradiation $-[\theta]_{222}$ in the dark) of the membrane composed of poly(L-glutamic acid) containing azobenzenesulfonate groups in the side chains at $25^{\circ} \mathrm{C}$.

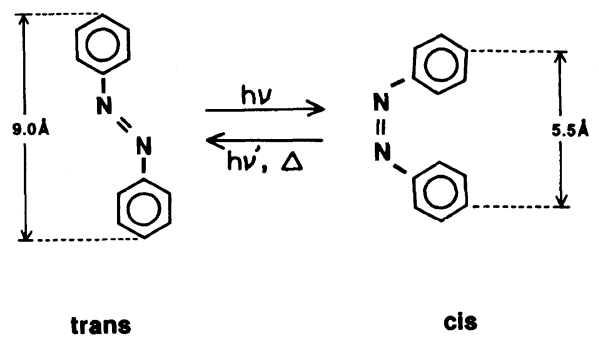

Scheme 3. 
around the partially charged helical rod at the adequate $\mathrm{pH}$ value, i.e., the increase in the electrostatic repulsion between sulfonate anions and carboxylate anions in the side chains resulting from a decrease in the distance between the para carbon atoms of the azobenzene moieties from $9.0 \AA$ to $5.5 \AA$ by light irradiation (Scheme 3). ${ }^{23}$

It is also found that the opposite conformational change of azo.S-14.1-PGA membrane from random coil to $\alpha$-helix could not be induced by irradiating at $\lambda>390 \mathrm{~nm}$ or by dark adaptation in the $\mathrm{pH}$ range where the UV light-induced $\alpha$-helix to coil transition occurs. This indicates that the isomerization of the azo chromophore from the cis to the trans form does not affect the conformation of the copolypeptide in the disordered structure.

\section{Photoinduced Membrane Potential Change of the Azo $S$-PGA Membrane}

The photocontrol of membrane potential across the polymer membrane system was first reported by J. Anzai et al., ${ }^{5}$ using an azobenzene-linked crown ether entrapped in poly(vinyl chloride) membrane as the photochromic molecule. They showed that the photoinduced membrane potential changes were due to changes in the binding ability of azo crown to potassium ions resulting from the trans to cis isomerization of the photochromic ionophore.

$\mathrm{We}^{15,17}$ also showed that the membrane potential across a membrane composed of PGA containing azobenzene in the side chains could be increased by photoisomerization, photoinduced polarity increase of the azobenzene side chain and induced dissociation of glutamic acid moiety. In this case, the photoinduced increase in the negative fixed charge density of the membrane was supported by the small increase in volume of the membrane, since the membrane structure, $\alpha$-helix form, was stable during irradiation. By using the azo S-PGA membrane, we extend the characterization of the photoinduced changes of

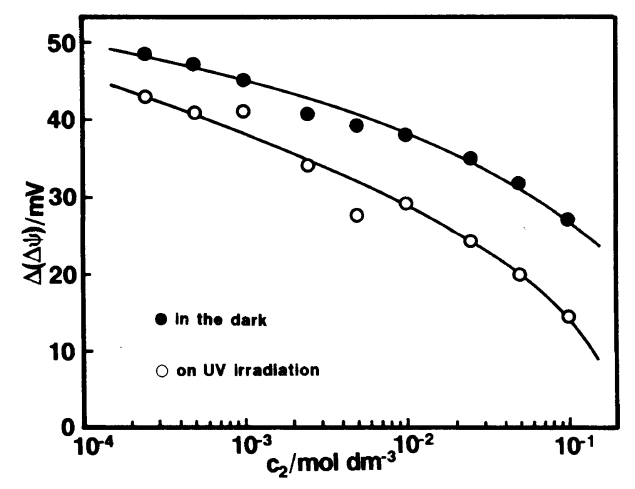

Figure 4. $\mathrm{KCl}$ concentration dependence of membrane potentials of the membrane composed of poly(Lglutamic acid) containing $14.1 \mathrm{~mol} \%$ azobenzenesulfonate groups in the side chains before UV irradiation (-O-) and after UV irradiation (-O-) at $\mathrm{pH} 7.0$ and at $25^{\circ} \mathrm{C}$.

membrane potential to the case in which the drastic structure change of the polypeptide membrane can be induced by the trans to cis isomerization of the azobenzene moieties.

Changes in the membrane potential, $\Delta(\Delta \psi)$, of the azo.S-14.1-PGA membrane on UV irradiation were observed with a $\mathrm{KCl}$ concentration gradient of $c_{1} / c_{2}=10$ at $25^{\circ} \mathrm{C}$. UV irradiation was carried out at $\mathrm{pH} 7.0$, since it was shown that the most remarkable conformational change of the azo-S-PGA membrane with $14.1 \mathrm{~mol} \%$ azobenzene sulfonate groups could be observed by irradiation at pH 7.0.

Figure 4 shows the concentration dependence of the membrane potential of the azo $\cdot \mathrm{S}$ 14.1-PGA membrane adapted in the dark or irradiated. UV irradiation was shown to induce a negative shift of the potential in the wide range of $\mathrm{KCl}$ concentration. The membrane potential, $\Delta(\Delta \psi)$, is the sum of the electrostatic potential (Donnan potential) differences between the membrane and aqueous solution on both sides of the membrane, $\Delta \psi_{D_{1}}$ and $\Delta \psi_{D_{2}}$, and the diffusion potential, $\Delta \psi_{\mathrm{d}}$. The Donnan potential is associated with the fixed charge density of the membrane and the diffusion potential is mainly concerned with the relative mobility of counter- and co-ions in 
the membrane phase, i.e., the transport number of ions in the membrane. $\Delta(\Delta \psi)$ of the negatively charged membrane is generally given as $^{24}$

\section{$\Delta(\Delta \psi)$}

$$
\begin{aligned}
= & \Delta \psi_{\mathrm{D}_{1}}+\Delta \psi_{\mathrm{D}_{2}}+\Delta \psi_{\mathrm{d}} \\
= & -\frac{R T}{F}\left[\ln \frac{c_{2}}{c_{1}}+\ln \left\{\frac{\sqrt{1+\left(\frac{2 K_{ \pm} c_{1}}{\phi X}\right)^{2}}+1}{\sqrt{1+\left(\frac{2 K_{ \pm} c_{2}}{\phi X}\right)^{2}}}\right\}\right. \\
& +\left(\frac{\bar{l}_{+}-\bar{l}_{-}}{\bar{l}_{+}+\bar{l}_{-}}\right) \ln \left\{\frac{\sqrt{1+\left(\frac{2 K_{ \pm} c_{2}}{\phi X}\right)^{2}}+\frac{\bar{l}_{+}-\bar{l}_{-}}{\bar{l}_{+}+\bar{l}_{-}}}{\sqrt{1+\left(\frac{2 K_{ \pm} c_{1}}{\phi X}\right)^{2}}+\frac{\bar{l}_{+}-\bar{l}_{-}}{\bar{l}_{+}+\bar{l}_{-}}}\right\}
\end{aligned}
$$

where $\bar{l}_{i}$ is the mobility of the $i$ th ion in the membrane, $\phi X$ is the effective (negative) charge density of the membrane, $K_{ \pm}$is the equilibrium partition coefficient, and $R, T$, $F$ are commonly used notations. We applied eq 1 to the experimental data in Figure 4. However, reasonable agreement between eq 1 and the experimental plots over the entire range of $\mathrm{KCl}$ concentration could not be obtained because of the fact that the membrane parameter, $\phi X$, varies with salt concentration, $c$, especially at low concentrations $(c \ll \phi X){ }^{25}$ So we applied eq 1 to the plots in Figure 4 in the limit range of high $\mathrm{KCl}$ concentration $\left(10^{-2} \mathrm{moll}^{-1}<c<10^{-1} \mathrm{moll}^{-1}\right)$ assuming that the concentration dependence of $\phi X$ is negligible. The curve fitting procedure, as a result, could produce the parameter corresponding to the effective charge density of the membrane, $\phi X / K_{ \pm}$, and transport numbers of $\mathrm{K}^{+}$and $\mathrm{Cl}^{-}$in the membrane, $t_{+}=\bar{l}_{+} /\left(\bar{l}_{+}+\bar{l}_{-}\right)$and $t_{-}=\bar{l}_{-} /\left(\bar{l}_{+}+\bar{l}_{-}\right)$, in the dark and irradiated state, respectively. .The results are shown in Table I. Irradiation induced a decrease in the value of the membrane parameter, $\phi X / K_{ \pm}$, of the membrane, i.e., the value of $\phi X / K_{ \pm}$of the irradiated membrane was one third that in the dark. The photoinduced decrease in the effective fixed charge concentration of the membrane in Table I implies an increase in the volume of the membrane system via photoinduced increase in the random coil content keeping the number of fixed charged groups constant.

Figure 5 shows the $\mathrm{pH}$-dependence of the degree of hydration, $\mathrm{H}$, of the azo.S-14.1PGA membrane in the dark. The value of $\mathrm{H}$ increased by increasing the $\mathrm{pH}$ of the aqueous solution. A comparison between the $\mathrm{pH}$ dependence of the backbone conformation (Figure 1(b)) and that of $H$ (Figure 5) indicates

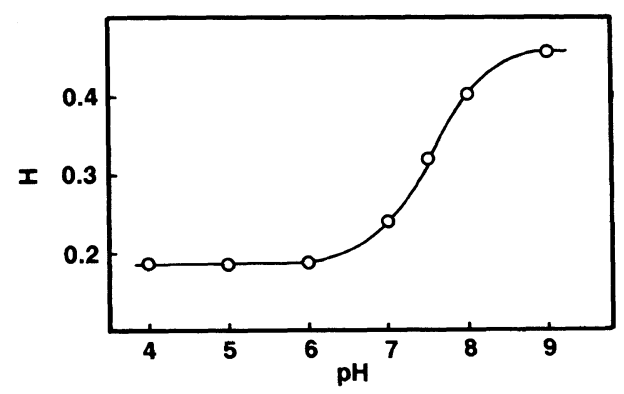

Figure 5. $\mathrm{pH}$ dependence of the degree of hydration, $H$, of the membrane composed of poly(L-glutamic acid) containing $14.1 \mathrm{~mol} \%$ azobenzenesulfonate groups in the side chains at $25^{\circ} \mathrm{C}$.

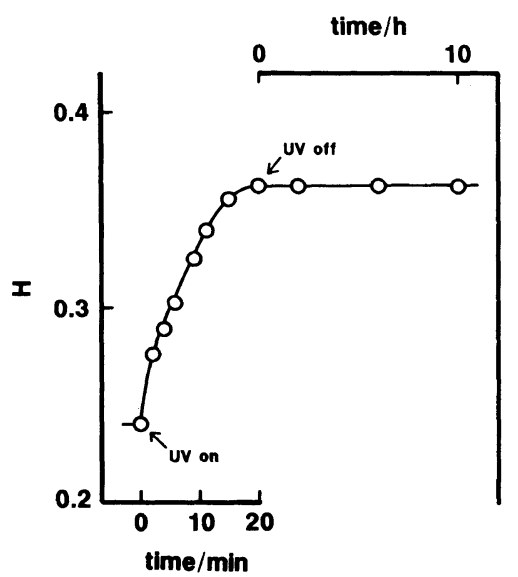

Figure 6. Changes in the degree of hydration, $H$, of the membrane composed of poly(L-glutamic acid) containing $14.1 \mathrm{~mol} \%$ azobenzenesulfonate groups in the side chains at $\mathrm{pH} 7.0$ and at $25^{\circ} \mathrm{C}$. 
Table I. Effect of UV irradiation on the fixed charge density, $\phi X / K_{ \pm}$, and transport numbers of $\mathrm{K}^{+}$and $\mathrm{Cl}^{-}\left(t_{+}\right.$and $t_{-}$, respectively), in azo $\cdot \mathrm{S}-14.1$ PGA membrane at $\mathrm{pH} 7.0$ and at $25^{\circ} \mathrm{C}$

\begin{tabular}{ccc}
\hline & In the dark & Irradiation \\
\hline$\phi X / K_{ \pm}$, mol dm $^{-3}$ & $2.58 \times 10^{-1}$ & $8.67 \times 10^{-2}$ \\
$t_{+}$ & 0.53 & 0.53 \\
$t_{-}$ & 0.47 & 0.47 \\
\hline
\end{tabular}

that the swelling of the membrane can occur based on increase in the content of random coil conformation, whose amido groups on the main chain are free to hydrate compared with that of $\alpha$-helix form. So we examined the changes in the degree of hydration of the azo.S-14.1-PGA membrane by the photoinduced helix to coil transition. Figure 6 shows the photoinduced changes in the $H$ value of the azo-S-14.1-PGA membrane when the $\mathrm{pH}$ of the aqueous solution was fixed at 7.0. As expected, the irradiation increased the degree of hydration of the membrane, and as a result, the value of $H$ increased from 0.24 to 0.36 by a factor of 1.5 resulting from the photoinduced helix to coil transition of the membrane (Figure 2(b)). The volume increase by a factor of 1.5 simply suggests that the effective fixed charge density, $\phi X$, of the membrane may decrease by a factor of 0.67 on light irradiation. On the other hand, the value of $\phi X / K_{ \pm}$of the irradiated membrane was shown to be one third the dark-adapted one (Table I). This means that the degree of the photoinduced decrease of the value of $\phi X / K_{ \pm}$, is slightly larger than that of $\phi X$. The one third decrease in the value of the membrane parameter, $\phi X / K_{ \pm}$, in Table I, therefore, is also attributable to the increase in the equilibrium partition coefficient, $K_{ \pm}$, based on the photoinduced swelling of the membrane in addition to the photoinduced decrease in the effective fixed charge density of the membrane.

On the other hand, the transport numbers in Table $\mathrm{I}, t_{+}$and $t_{-}$, are shown to be independent of UV irradiation. It is concluded, therefore, that the photoinduced membrane potential change is a result of changes in the effective fixed charge density and equilibrium partition coefficient of the membrane, i.e., changes in Donnan potential, $\Delta \psi_{\mathrm{D}}$ terms in eq 1 , via change in the membrane structure from $\alpha$-helix to coil with UV irradiation.

It was also found that after removal of the light, dark adaptation did not induce any change in the membrane potential and $H$ value, nor in the irreversibility of the photoinduced conformational transition.

Acknowledgement. The authors gratefully acknowledge the support of the Japanese Ministry of Education through a Grant-in-Aid for Developmental Science Research.

\section{REFERENCES}

1. S. Kato, M. Aizawa, and S. Suzuki, J. Membrane Sci., 1, 289 (1976).

2. S. Kato, M. Aizawa, and S. Suzuki, J. Membrane Sci., 2, 39 (1977).

3. M. Aizawa, S. Tomono, and S. Suzuki, J. Membrane Sci., 2, 289 (1977).

4. S. Kato, M. Aizawa, and S. Suzuki, Kobunshi Ronbunshu, 34, 793 (1977).

5. J. Anzai, H. Sasaki, A. Ueno, and T. Osa, J. Chem. Soc., Chem. Commun., 1045 (1976).

6. H. Sasaki, A. Ueno, J. Anzai, and T. Osa, Bull. Chem. Soc. Jpn., 59, 1953 (1986).

7. H. Sasaki, A. Ueno, and T. Osa, Chem. Lett., 529 (1987).

8. J. Anzai, Y. Hasebe, A. Ueno, and T. Osa, Polym. Prepr., Jpn., 37, 2881 (1988).

9. M. Irie, A. Menju, and K. Hayashi, Nippon Kagaku Kaishi, 227 (1984).

10. F. Ciardelli, O. Pieroni, A. Fissi, and J. L. Houben, Biopolymers, 23, 1423 (1984).

11. A. Fissi, O. Pieroni, and F. Ciardelli, Biopolymers, 26, 1993 (1987).

12. A. Ueno, J. Anzai, T. Osa, and Y. Kadoma, J. Polym. Sci., Polym. Lett. Ed., 15, 407 (1977).

13. A. Ueno, J. Anzai, K. Takahashi, and T. Osa, Kobunshi Ronbunshu, 37, 281 (1980).

14. A. Ueno, K. Takahashị, J. Anzai, and T. Osa, Mackromol. Chem., 182, 693 (1981).

15. A. Takizawa, M. Sato, T. Kinoshita, and Y. Tsujita, Chem. Lett., 1963 (1984).

16. T. Kinoshita, M. Sato, A. Takizawa, and Y. Tsujita, J. Chem. Soc., Chem. Commun., 929 (1984). 
17. T. Kinoshita, M. Sato, A. Takizawa, and Y. Tsujita, Macromolecules, 19, 51 (1986).

18. T. Kinoshita, M. Sato, A. Takizawa, and Y. Tsujita, J. Am. Chem. Soc., 108, 6399 (1986).

19. Y. Sato, T. Kinoshita, A. Takizawa, and Y. Tsujita, Macromolecules, 21, 3419 (1988).

20. M. Sato, T. Kinoshita, A. Takizawa, and Y. Tsujita, Polym. J., 20, 729 (1988).

21. M. Sato, T. Kinoshita, A. Takizawa, and Y. Tsujita,
Macromolecules, 21, 1612 (1988).

22. M. Sato, T. Kinoshita, A. Takizawa, and Y. Tsujita, Polym. J., 20, 761 (1988).

23. G. C. Hampson and J. M. Robertson, J. Chem. Soc., 401 (1941).

24. A. Takizawa, Hyomen, 20, 68 (1982).

25. M. Tasaka, N. Aoki, Y. Kondo, and M. Nagasawa, J. Phys. Chem., 79, 1307 (1975). 